\title{
Withaferin-A Inhibits Colon Cancer Cell Growth by Blocking STAT3 Transcriptional Activity
}

\author{
Bu Young Choi ${ }^{1}$, Bong-Woo Kim ${ }^{2}$ \\ 'Department of Pharmaceutical Science and Engineering, ${ }^{2}$ Department of Cosmetic Science and Technology, Seowon University, Cheongju, Korea
}

\begin{abstract}
Background: Withania somnifera (known as Ashwagandha) is a medicinal plant used in the ayurvedic medicines in India. Withaferin- $\mathrm{A}$, a withanolide derived from the leaf extract of $W$. somnifera, has been reported to exhibit anti-tumor activity against various cancer cells, such as leukemia, breast cancer and colon cancer cells.

Methods: We investigated the anti-cancer effects of withaferin-A on the proliferation and migration of human colorectal cancer (HCT116) cells. And we evaluated the effects of withaferin-A on the transcriptional activity of STAT3 and the growth of HCT116 cells in xenograft mouse tumor model.

Results: In the present study, we found that withaferin-A inhibited the proliferation and migration of HCT116 cells in a concentration-dependent manner. Treatment of HCT116 cells with withaferin-A attenuated interleukin-6-induced activation of STAT3, which has been implicated in the development and progression of colon cancer. To examine the effect of withaferin-A on HCT116 cells proliferation in vivo, we generated HCT116 cells xenograft tumors in Balb/c nude mice and treated the tumor bearing mice with or without withaferin-A intraperitoneally. Treatment with withaferin-A exhibited significant decrease in the volume and weight of tumors as compared to untreated controls.
\end{abstract}

Conclusions: The present study suggests that withaferin-A holds the potential to be developed as a small molecule inhibitor of STAT3 for the treatment of HCT116.

(J Cancer Prev 2015;20:185-192)

Key Words: Withaferin-A, Colorectal neoplasms, STAT3 transcription factor, Heterografts

\section{INTRODUCTION}

Colorectal cancer (CRC) is one of the most common cancers in the world. ${ }^{1}$ According to global cancer statistics, CRC appears as the fourth leading cause of cancer-related deaths worldwide. ${ }^{2}$ Although remarkable progress has been made in developing effective chemotherapeutic agents to treat early-stage CRC, emerging incidences of drug resistance often leads to chemotherapy failure, and impose high toxicity to bone marrow, gastrointestinal tract, and skin. ${ }^{3}$ Therefore, there is an increasing interest in searching for new treatment and/or prevention modalities for CRC.

In recent years, bioactive plant constituents have got much attention for the development of new therapeutic agents. A wide variety of phytochemicals are being screened for their anticancer potential. Withaferin-A (Fig. 1A), originally isolated from Withania somnifera, has long been used as traditional medicine for the treatment of various cancers. ${ }^{4.5} \mathrm{~A}$ good deal of research has already been done with withaferin-A as a potential cancer therapy over the last few years. For example, withaferin-A suppressed tumor growth through various mechanisms including reactive oxygen species (ROS) generation, proteasome inhibition, p53 stabilization, induction of endoplasmic reticulum stress, inhibition of Akt phosphorylation, and p38 MAPK activation. ${ }^{6-11}$ Withaferin-A also induced apoptosis in human breast cancer (MCF-7 and MDA-231) cells through the inhibition of Notch

Received August 5, 2015, Revised August 18, 2015, Accepted August 18, 2015

Correspondence to: Bong-Woo Kim

Department of Cosmetic Science and Technology, Seowon University, 377-3 Musimseo-ro, Seowon-gu, Cheongju 28674, Korea

Tel: +82-43-299-8493, Fax: +82-43-299-8470, E-mail: kbw@seowon.ac.kr, ORCID: Bong-Woo Kim, http://orcid.org/0000-0001-8767-9519

Copyright (C) 2015 Korean Society of Cancer Prevention

(c) This is an Open Access article distributed under the terms of the Creative Commons Attribution Non-Commercial License (http://creativecommons.org/licenses/by-nc/4.0) which permits unrestricted non-commercial use, distribution, and reproduction in any medium, provided the original work is properly cited. 
A

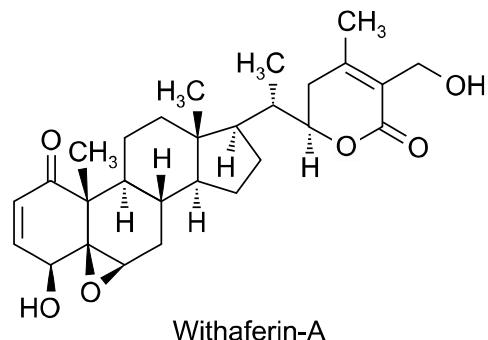
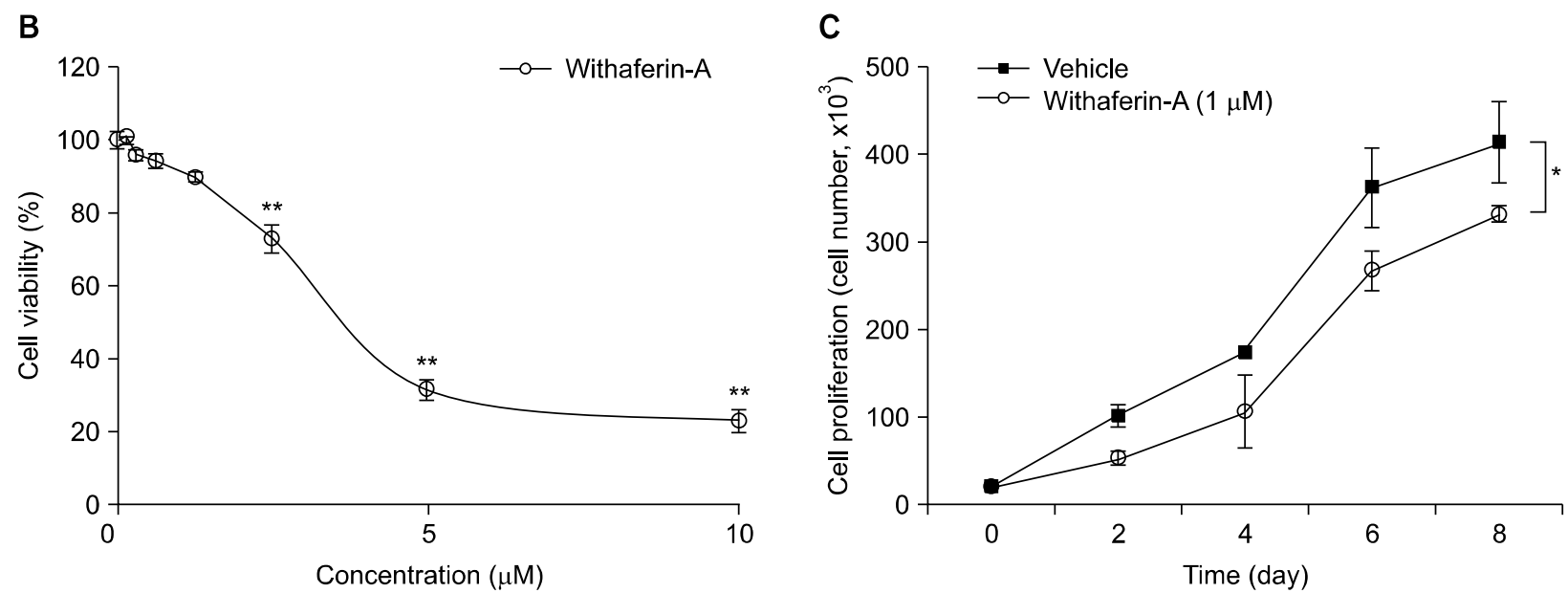

Figure 1. Effect of withaferin A on the proliferation of human colorectal cancer (HCT116) cells. (A) The structure of withaferin-A. (B) Cell viability was analyzed using MTT cell proliferation assay kits. The proliferation of HCT116 cells treated with withaferin-A was significantly decreased in a concentration-dependent manner. (C) Cell proliferation pattern of HCT116 cells was measured by counting cells after exposure to $1 \mu \mathrm{M}$ withaferin-A during 8 days. At the last day of exposure, cells treated with withaferin-A $(1 \mu \mathrm{M})$ showed a dramatic decrease in cell proliferation when compared with the vehicle group. All data are presented as the means $\pm \mathrm{SE}$; $t$-test and ANOVA. $* P<0.05$ and $* * P<0.01$

signaling cascades. ${ }^{12}$ More recent studies demonstrated that withaferin-A induced apoptosis in human renal carcinoma (Caki) cells ${ }^{13,14}$ and inhibited the growth of neuroblastoma and multiple myeloma cells. ${ }^{15}$ The present study was designed to examine the effect of withaferin-A in CRC and to elucidate its underlying mechanisms.

Tumor promotion and progression involve inappropriately increased cell proliferation, evasion from apoptosis, induction of tumor angiogenesis, and increased migration, invasion and metastasis of cancer cells. Among various oncogenic signaling pathways, STAT3 plays a crucial role in CRC. ${ }^{16}$ It has been reported that persistent STAT3 activation enhances the proliferation of colon cancer cells in culture and the growth xenograft tumors of these cells when implanted in nude mice. ${ }^{17}$ Moreover, the blockade of STAT3 signaling led colon cancer cells to undergo apoptosis. ${ }^{18}$ Since abnormal activation of STAT3 signaling induces transcriptional activation the genes encoding various proteins involved in apoptosis, cell cycle progression, angiogenesis, invasion and metastasis, targeted inhibition of STAT3 signaling is a pragmatic approach in preventing CRC. ${ }^{19,20}$ Therefore, many studies are investigated to develop new therapeutic agents that are able to directly inhibit the activation of STAT3 signaling. ${ }^{21.22}$ In this study, we have investigated the effects of withaferin-A on the proliferation and migration of human colorectal cancer (HCT116) cells and the growth of these cell's xenograft tumors in nude mice. Here, we report that withaferin-A inhibited the proliferation, migration and xenograft tumor growth of HCT116 cells by suppressing the STAT3 transcriptional activity in HCT116 cells.

\section{MATERIALS AND METHODS}

\section{Chemicals and reagents}

Withaferin-A was purchased from Sigma Aldrich (St. Louis, MO, USA). Withaferin-A was dissolved in methanol at a stock concentration of $10 \mathrm{mM}$ and diluted with medium prior to experimental procedures (final concentration of methanol $\leq$ 0.01\%). Methylthiazolyldiphenyl-tetrazolium bromide (MTT) and paraformaldehyde was purchased from Sigma Aldrich. Primary 
antibody for interleukin-6 (IL-6) was from R\&D Systems (Minneapolis, MN, USA) and that of proliferating cell nuclear antigen (PCNA) and secondary antibodies were from Cell signaling Technology (Beverly, MA, USA).

\section{Cell culture}

HCT116 cells (ATCC, Rockville, MD, USA) were grown in the RPMI-1640 medium (Gibco ${ }^{\circledR}$; Life Technologies, Grand Island, NY, USA) supplemented with $10 \% \mathrm{FBS}, 100 \mathrm{U} / \mathrm{mL}$ penicillin and streptomycin. Cells were maintained in an incubator at $37^{\circ} \mathrm{C}$ in $5 \% \mathrm{CO}_{2}$. For the treatment of cells with withaferin-A, cells were cultured in RPMI-1640 medium without FBS for 12 hours.

\section{Cell viability assay}

Cells were plated at a density of $1 \times 10^{4}$ cells per well into 96-well plates and maintained at $37^{\circ} \mathrm{C}$ for 24 hours. Cells were treated with various concentrations of withaferin-A for 12 hours. Fresh medium containing $50 \mu \mathrm{L}$ of MTT solution $(0.5 \mathrm{mg} / \mathrm{mL})$ was then added to each well. After incubation for another 3 hours, the MTT formazan crystals were dissolved in dimethyl sulfoxide (DMSO) and viable cells were detected by measuring the absorbance at $570 \mathrm{~nm}$ using a microplate reader (Molecular Devices, Sunnyvale, CA, USA).

\section{Wound-healing assay}

For a scratch wound healing assay, HCT116 cells were subcultured in 12 -well plates and grown to $70 \%$ to $80 \%$ confluence. Monolayer of cells was scratched with a sterile $10-\mu \mathrm{L}$ micropipette tip across center of the well. Cells were washed 3 times with PBS to remove cellular debris, and were exposed to medium containing withaferin-A. Cell migration was observed under a bright-field microscope (Olympus, Tokyo, Japan) at a magnification of $100 \times$. The migration velocity was calculated independently in five randomly chosen fields of 6 scratches, and the percentage of inhibition was shown using control cells at $100 \%$.

\section{STAT3 transcription factor assay}

STAT3 luciferase-reporter gene assay was performed as described previously. ${ }^{23}$ Briefly, STAT3-luc constructs were stably transfected into HCT116 cells. Cells were plated at a density of $2 \times 10^{4}$ cells per well in 96-well plate in RPMI medium and treated with IL-6, withaferin-A or methanol (control). After incubation for another 12 hours, cells were lysed by passive lysis buffer and incubated for 15 minutes in orbital shaker. The luciferase activity was measured by dual-luciferase reporter assay kit (Promega, Madison, WI, USA) according to the manufacturer's protocol.

\section{Immunofluorescence staining}

HCT116 cells were fixed with 3.7\% paraformaldehyde for 10 minutes and then permeabilized with 0.1\% Triton X-100 (Sigma Aldrich). The cells were blocked for 1 hour with blocking buffer ( $5 \%$ bovine serum albumin in PBS). The subsequent incubations with primary antibodies and fluorescent-conjugated secondary antibodies were conducted at room temperature. Immunofluorescence images were captured by a laser scanning confocal microscope (ZEISS LSM 510 META; Carl Zeiss, Oberkoden, Germany).

\section{Tumor xenograft models}

HCT116 cells $\left(6 \times 10^{6}\right.$ cells/200 $\left.\mu \mathrm{L}\right)$ were subcutaneously injected into the flank of the female athymic BALB/C nude mice at the age of 6 weeks. Seven days after the injection of cells, mice were randomized into treatment groups and control groups. The treatment groups were injected with withaferin-A $(2 \mathrm{mg} / \mathrm{kg}$ of body weight, DMSO $0.1 \% \mathrm{v} / \mathrm{v}$ ) every 2 days and the control groups were injected with vehicle (DMSO $0.1 \% \mathrm{v} / \mathrm{v}$ ), intraperitoneally. Tumor diameters were measured 3 times a week, and tumor volumes were calculated according to the following formula (length $\times$ width $^{2} \times \pi / 6$ ). After 32 days of treatment, the mice were sacrificed and the tumors were harvested, weighted, and fixed in fix solution (4\% paraformaldehyde) for immunohistochemistry.

\section{Statistical analysis}

Statistical analysis was performed by either two-tailed Student's $t$-test or ANOVA test with post $t$-test using GraphPad InStat (GraphPad Software, San Diego, CA, USA). P-value less than 0.05 was considered as significant.

\section{RESULTS}

\section{Withaferin-A inhibits the proliferation of HCT116 cells}

To investigate whether withaferin-A inhibits the proliferation and growth of HCT116 cells in culture, cells were treated with varying concentrations of withaferin-A for indicated periods and the levels of proliferation were measured. As shown in Figure 1, the MTT assay and cell counting revealed that withaferin-A significantly decreased the viability of HCT116 cells in concentration- and time-dependent manner. Cell viability was significantly decreased by treatment with withaferin- $\mathrm{A}$ at concentrations below $5 \mu \mathrm{M}$, showing $69 \%$ reduction in the proliferation of HCT116 cells after 12 hour (Fig. 1B). To assess the effect of withaferin-A on the proliferation of HCT116 cells, we counted the 
cell at 2, 4, 6, and 8 days after treatment with $1 \mu \mathrm{M}$ withaferin-A. At the last day of treatment, the cells showed a dramatic decrease in cell proliferation when compared with the vehicle group (Fig. 1C).

\section{Withaferin-A inhibits the migration of HCT116 cells}

Since tumor cell migration is a critical step in the invasion and metastasis of cancer, we examined the effect of withaferin-A on the migration ability of HCT116 cells. Confluent cells were scraped across center of the well and the remaining cells were incubated to migrate into the gap in the absence or presence of withaferin-A (Fig. 2A). After 24 hours incubation, the wound gap was wider in the withaferin-A-treated groups $(0.001-1 \mu \mathrm{M})$ as compared to the untreated group, indicating that withaferin-A inhibits the motility of HCT116 cells (Fig. 2A and 2B). Next, we tested the effects of withaferin-A on cell migration by the Transwell chamber assay. As shown in Figure 2C, treatment with withaferin-A $(1 \mu \mathrm{M})$ significantly reduced cell migration measured as percent of control (Fig. 2D).

\section{Inhibition of interleukin-6-induced STAT3 transcrip- tional activity by withaferin-A in HCT116 cells}

Since inappropriate induction of a variety of STAT3 target genes has been implicated in the development of CRC, we assessed the effects of withaferin-A on the transcriptional activity of STAT3. HCT116 cells were stably transfected with STAT3-luc constructs, and incubated with various concentrations $(0.0625$,
A

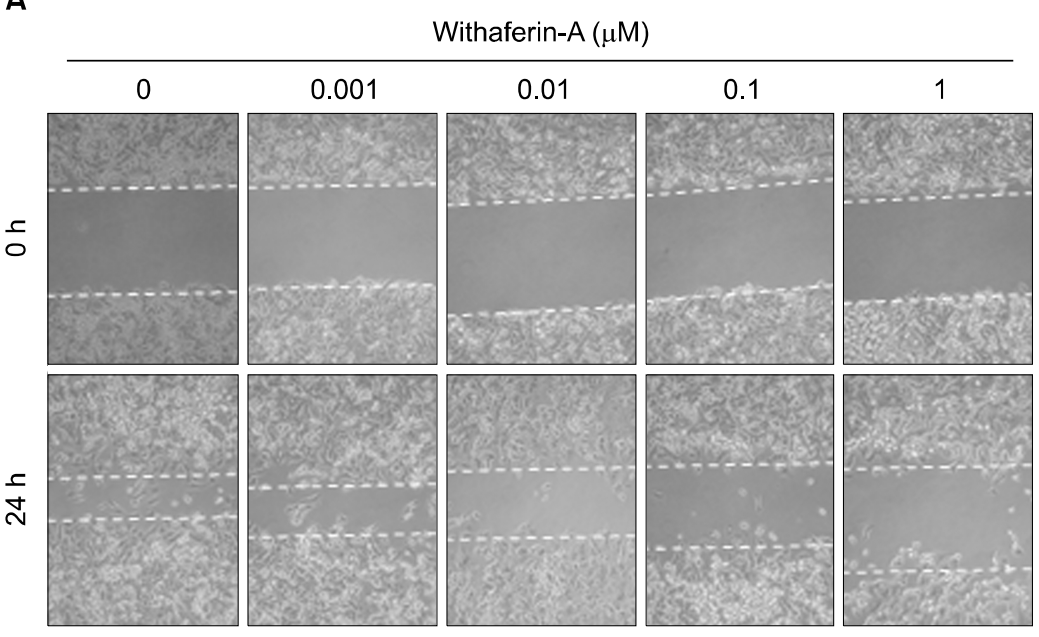

C

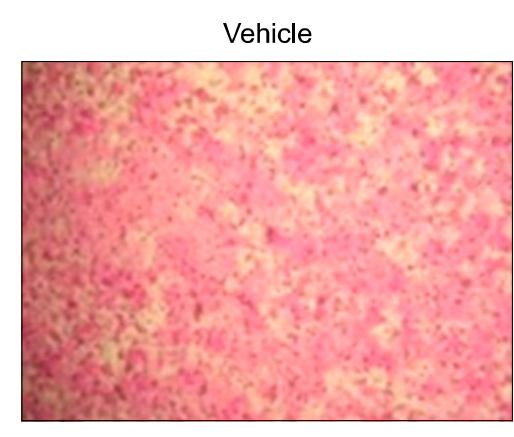

B

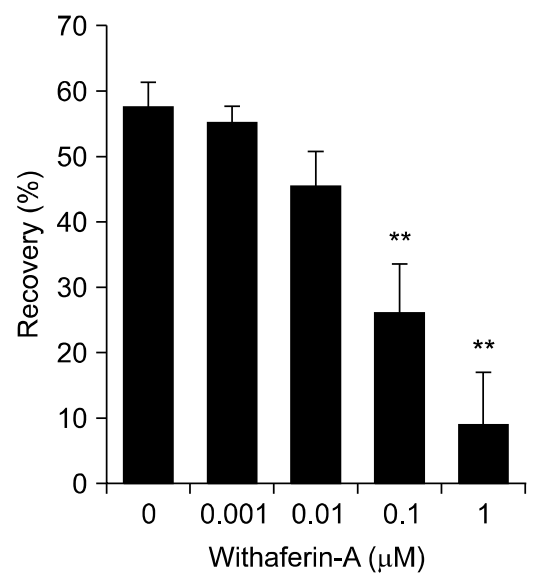

D

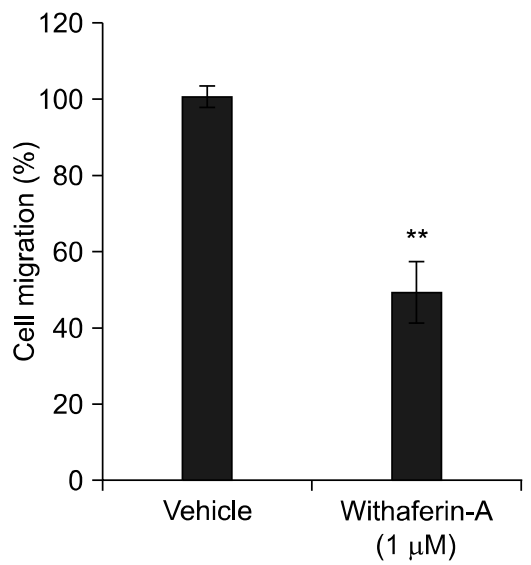

Figure 2. Effect of withaferin-A on the migration of human colorectal cancer (HCT116) cells. (A, B) Cells were treated with or without withaferin-A, and subjected to wound-healing assay as described in materials and methods. The area of the wound was measured at 0 hour and 24 hours. The wound recovery was calculated and compared between untreated cells with that of withaferin-A-treated cells. The results of migrated cells were representative of three experiment each done in triplicate (A: $\times 20)$. (C, D) HCT116 cells were treated with 0 or $1 \mu \mathrm{M}$ withaferin-A and $0.01 \%$ methanol (vehicle) for 48 hours and subjected to Transwell migration assay (C: Eosin staining, $\times 200$ ). Results represent three independent experiments and each experiment was done in triplicate. All data are presented as the means $\pm \mathrm{SE}$; $t$-test and ANOVA. $* * P<0.01$. 
$0.125,0.5$, or $1 \mu \mathrm{M}$ ) of withaferin-A. As shown in Figure 3, incubation of cells with IL-6 induced the STAT3 reporter gene activity, which was blunted by treatment of cells with withaferin$\mathrm{A}$ in a concentration-dependent manner. And, we assessed whether withaferin-A treatment induces nuclear translocation of STAT3. Immunofluorescence staining showed that IL-6-induced nuclear distribution of STAT3 in HCT116 cells significantly reduced by after treatment with $1 \mu \mathrm{M}$ withaferin-A.

\section{Withaferin-A attenuates the growth of HCT116 cells xenograft tumor in nude mice}

We next evaluated the effect of withaferin-A on the growth of
HCT116 cells in vivo by using a xenograft mouse tumor model. Athymic BALB/C nude mice were subcutaneously injected with HCT116 cells to generate xenograft tumors. Intraperitoneal administration of withaferin-A every 2 days for 32 days led to a significant decrease in the volume and weight of tumors as compared to untreated controls without affecting the mean body weight (Fig. 4). The mean tumor volume of withaferin-A-treated mice was 404.5 $\pm 67.6 \mathrm{~mm}^{3}$, which was approximately 1.44 fold lower compared with that in control mice $\left(582.6 \pm 42.6 \mathrm{~mm}^{3}, P=0.017\right)$ (Fig. 4B and $4 \mathrm{C}$ ). As shown in Figure $4 \mathrm{C}$, the average tumor weight was also significantly reduced by the treatment with withaferin-A. Immunohistochemical staining of sections of xenograft tumors
A

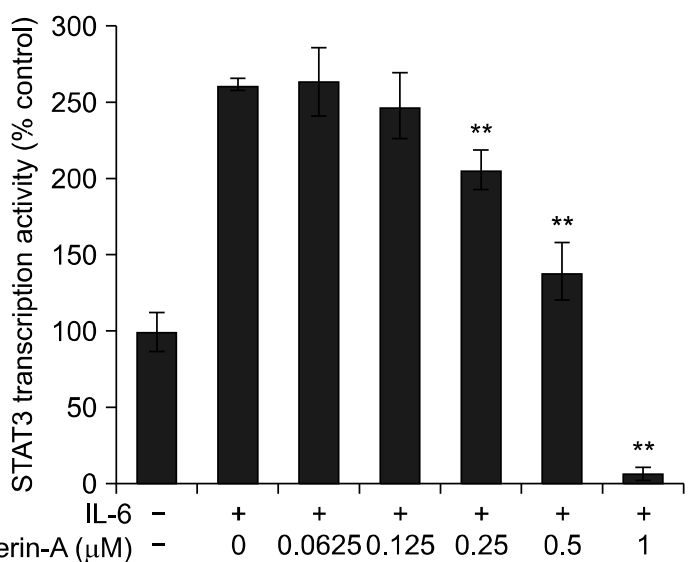

C

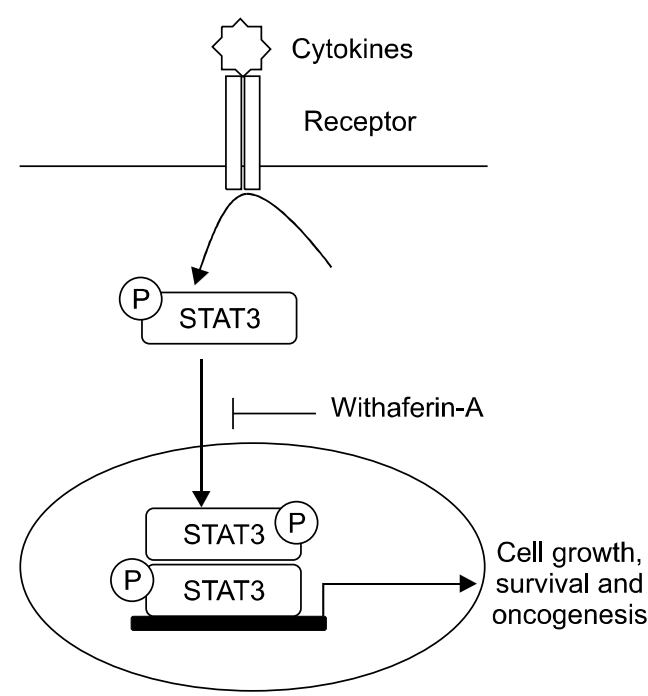

B

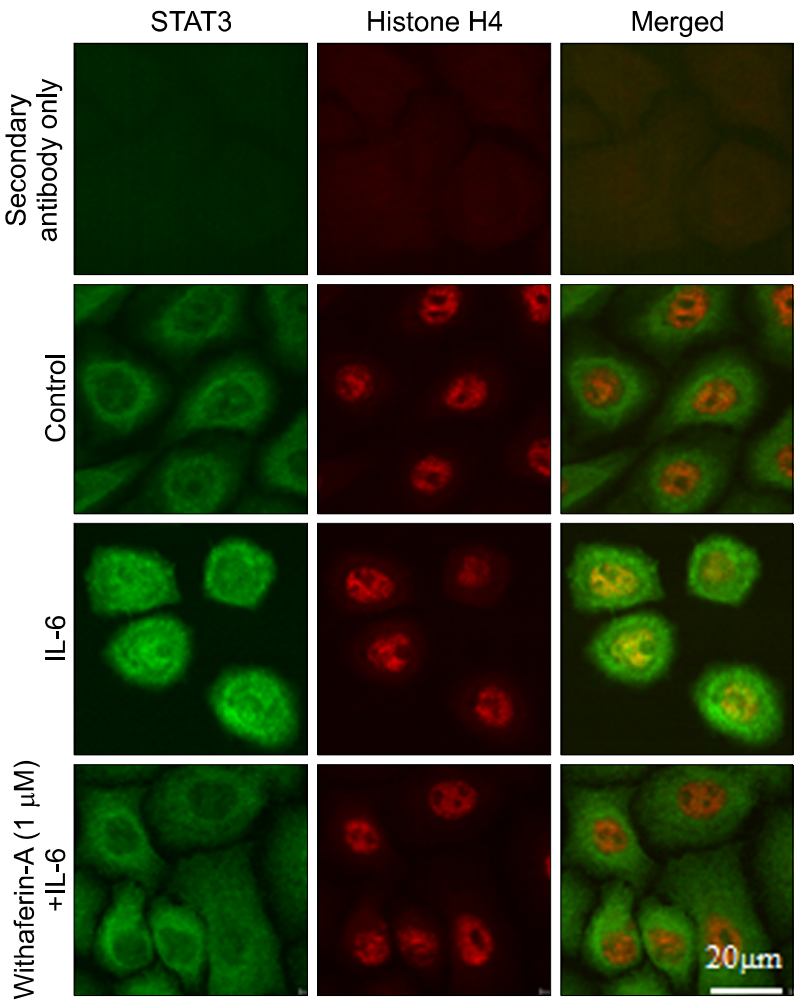

Figure 3. Effect of withaferin-A on interleukin-6 (IL-6)-induced STAT3 transcriptional activation. (A) STAT3 transcriptional activity assay using the nuclear extract from HCT116 cells treated with the indicated concentrations of withaferin-A with or without IL-6 (10 ng/mL) stimulation for 12 hours. Results are representative of three individual experiments. All data are presented as the means \pm SE; $t$-test and ANOVA. $* * P<0.01$. (B) The nuclear translocation of STAT3 in HCT116 cells after treatment with IL-6 $(10 \mathrm{ng} / \mathrm{mL})$ was inhibited by withaferin-A. For immunofluorescence staining, cells were permeabilized and stained with anti-STAT-3 antibody after fixation (green). Anti-histone H4 antibody was used to stain the cell nuclei B (red). (C) A schematic model showing a role of withaferin-A in the modulation of STAT3 signaling. 
A

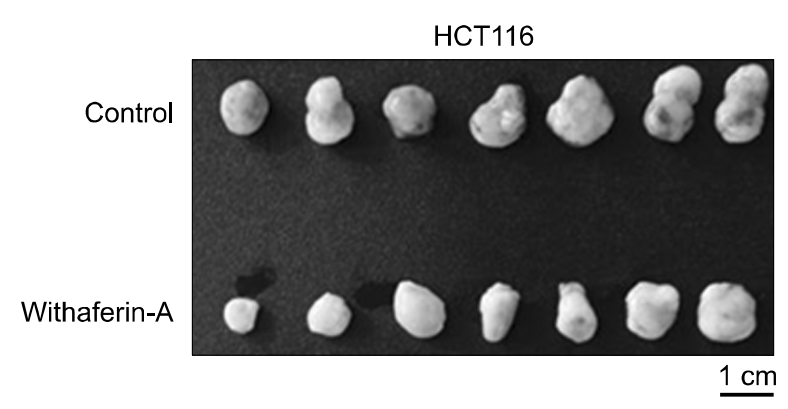

C

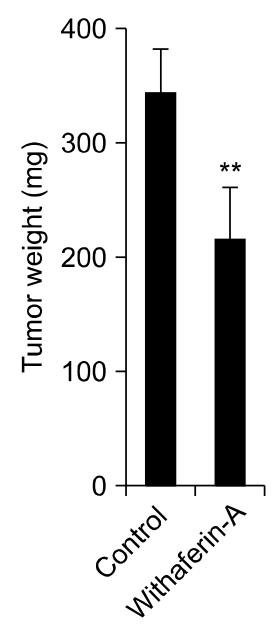

D

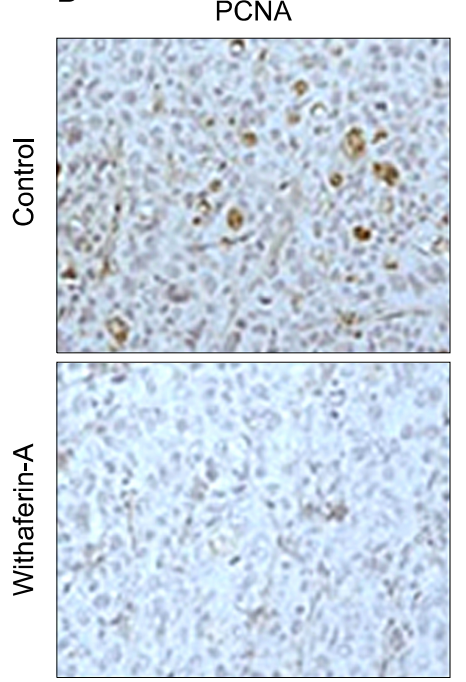

B

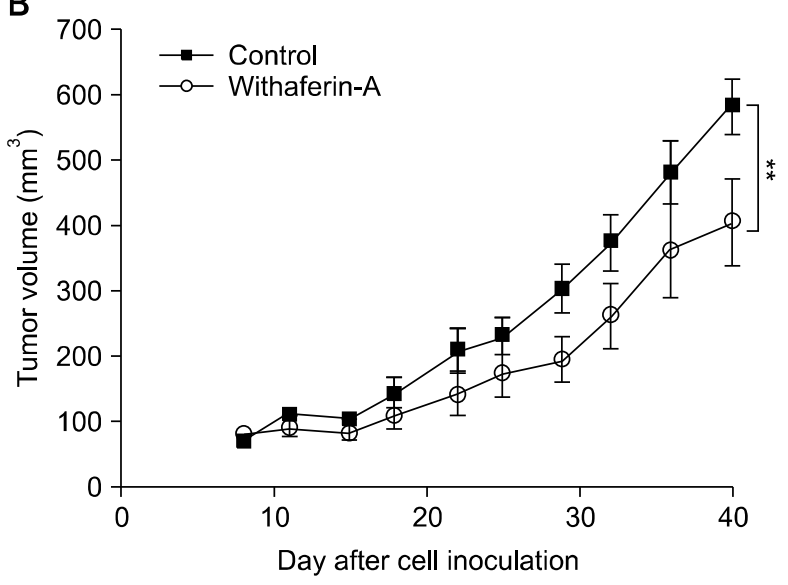

$\mathrm{E}$

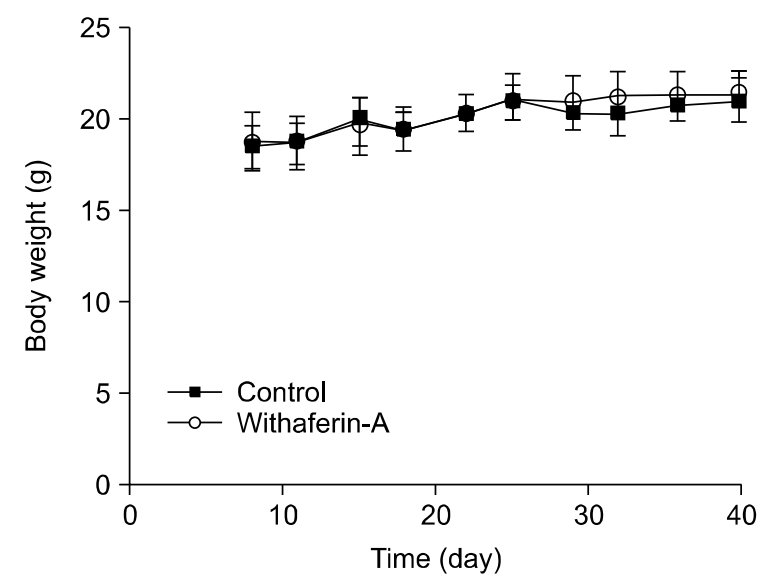

Figure 4. Effect of withaferin-A on the growth of human colorectal cancer (HCT116) cells tumor xenograft in nude mice. (A) HCT116 cells were subcutaneously injected into the flanks of athymic BALB/C nude mice. At 1 week post tumor cell injection, withaferin-A was administered by intraperitoneal injection every 2 days for 32 days. Representative images of the retrieved xenograft tumors at the endpoint. (B) Tumor volumes were measured with a caliper every 2 nd day. Significant decrease in tumor size was observed in mice treated with withaferin-A as compared to those of control mice. (C) The average weight of the tumors from mice was analyzed. (D) For tumor cell proliferation analysis, sections of xenograft tumors from both withaferin-A-treated and untreated animals were subjected to immunohistochemical stating with an anti-proliferating cell nuclear antigen (PCNA) antibody $(\times 20)$. (E) The corresponding body weight changes during the withaferin-A treatments. All data are presented as the means $\pm \mathrm{SE}$; $t$-test and ANOVA. $* * P<0.01$.

from mice treated with withaferin-A or untreated controls showed that withaferin-A treatment caused a marked inhibition of the expression of PCNA, which is a marker of tumor cell proliferation (Fig. 4D).

\section{DISCUSSION}

Plant secondary metabolites always remain in the front line in the development of new therapeutic agents. In the context of emerging evidence of chemotherapy failure and growing trend of chemo-resistance, bioactive phytochemicals are given much importance for searching new anticancer therapeutics. Numerous preclinical studies have demonstrated the anticancer effects of a wide variety of plant polyphenols. Withaferin-A has been extensively investigated for its diverse pharmacological activities. ${ }^{24}$ Although the anticancer effects of withaferin-A has been reported in various preclinical studies, ${ }^{15.25 .26}$ its molecular mechanism of action remains elusive. Our study has revealed that withaferin-A decreased the viability of HCT116 cells in a time- and concentrationdependent manner. It has also been reported earlier that withaferin-A induces apoptosis in various colon cancer cells including HCT116 cells by blocking Notch1-mediated prosurvival 
signaling pathways. ${ }^{27}$ However, withaferin-A had no effect of cell viabilities in androgen sensitive normal human fibroblasts and prostate adenocarcinoma cells indicating that withaferin-A induces selective tumor death. ${ }^{28}$ Our study also revealed that withaferin-A inhibited the proliferation of HCT116 cells.

Previous studies have demonstrated that withaferin-A induces apoptosis in various cancer cells by multiple mechanisms including the generation of ROS, mitochondrial dysfunction, cell cycle arrest, inactivation of prosurvival signaling molecules, such as extracellular signal-regulated kinase, c-Jun $\mathrm{N}$ terminal kinase, NF-KB and STAT3. ${ }^{6,11,13,29}$ These prosurvival factors are also involved in the enhanced migration of cancer cells. Our findings that withaferin-A inhibited migration of HCT116 cells suggests that the compound might interfere with the cell signaling pathways those are involved in increased proliferation and migration of cancer cells.

One of the major oncogenic signaling pathways is the STAT3 signaling. The inappropriate activation of STAT3 contributes to the survival, proliferation, chemo-resistance, and metastasis of cancer cell, and is constitutively overexpressed in various cancer cells such as sarcoma, lymphoma, carcinoma and leukemia. ${ }^{30}$ It has been reported that STAT3 activation increased the rate of proliferation and growth of colon cancer cells, ${ }^{17}$ while inactivation of these gene induces apoptosis. ${ }^{18}$ Therefore, many small molecules have been discovered to directly inhibit STAT3 activation. Although several small molecules such as alantoactone, resveratrol and fluacrypyrim inhibit STAT3 signaling, most of these molecules block the STAT3 signaling by suppress STAT3 upstream kinases indirectly. ${ }^{21-23}$ However, withaferin-A was reported to cause direct inhibition of STAT3 and induction of apoptotic cell death in various cancer cells. s.31,32 $^{3}$

The activation of STAT3 is mediated through the phosphorylation of its tyrosine-705 residue followed by the formation of STAT3 dimer, ${ }^{33}$ which then translocates to the nucleus and binds to the gamma activated sites of genes encoding proteins engaged in increased cell proliferation and migration. These genes products include cell cycle regulatory proteins (e.g., cyclins and cyclin-dependent kinases), antiapoptotic proteins (e.g., Bcl-2, BCl-xl), and cell proliferation markers (e.g., PCNA and survivin). ${ }^{34}$ The phosphorylation of STAT3 at tyrosine-705 residue is mediated by the upstream kinases, such as JAK2. ${ }^{35}$ In a recent study, Yco et al. ${ }^{15}$ demonstrated that withaferin-A inhibited STAT3 phosphorylation, thereby blocked STAT3 dimerization by directly binding to the STAT3 Src homology (SH2). Our findings that withaferin-A attenuated the transcriptional activity of STAT3 in IL-6 stimulated HCT116 cells were in good correlation with the report of Um et al., ${ }^{13}$ who demonstrated that withaferin-A diminished IL-6-induced phosphorylation of JAK2 and STAT3 in renal carcinoma cells. Further in vivo experimental evidence of decreased xenograft tumor growth of HCT116 cells and the reduced expression of PCNA in tumor tissues upon administration of withaferin-A suggest the potential of this compound to be used for the prevention and/or therapy of cancer. In conclusion, withaferin-A is able to inhibit not only the proliferation of HCT116 cells but also attenuated the tumor growth in vivo by suppressing STAT3 signaling pathways.

\section{CONFLICTS OF INTEREST}

No potential conflicts of interest were disclosed.

\section{REFERENCES}

1. Jemal A, Siegel R, Ward E, Hao Y, Xu J, Thun MJ. Cancer statistics, 2009. CA Cancer J Clin 2009:59:225-49.

2. Ferlay J, Shin HR, Bray F, Forman D, Mathers C, Parkin DM. Estimates of worldwide burden of cancer in 2008: GLOBOCAN 2008. Int J Cancer 2010;127:2893-917.

3. Gusella M, Frigo AC, Bolzonella C, Marinelli R, Barile C, Bononi A, et al. Predictors of survival and toxicity in patients on adjuvant therapy with 5-fluorouracil for colorectal cancer. Br J Cancer 2009;100:1549-57.

4. Bhattacharya SK, Satyan KS, Ghosal S. Antioxidant activity of glycowithanolides from Withania somnifera. Indian J Exp Biol 1997;35:236-9.

5. Mohan R, Hammers HJ, Bargagna-Mohan P, Zhan XH, Herbstritt $\mathrm{CJ}$, Ruiz A, et al. Withaferin A is a potent inhibitor of angiogenesis. Angiogenesis 2004;7:115-22.

6. Malik F, Kumar A, Bhushan S, Khan S, Bhatia A, Suri KA, et al. Reactive oxygen species generation and mitochondrial dysfunction in the apoptotic cell death of human myeloid leukemia HL-60 cells by a dietary compound withaferin A with concomitant protection by $\mathrm{N}$-acetyl cysteine. Apoptosis 2007;12: 2115-33.

7. Yang H, Shi G, Dou QP. The tumor proteasome is a primary target for the natural anticancer compound Withaferin A isolated from "Indian winter cherry". Mol Pharmacol 2007;71:426-37.

8. Munagala R, Kausar H, Munjal C, Gupta RC. Withaferin A induces p53-dependent apoptosis by repression of HPV oncogenes and upregulation of tumor suppressor proteins in human cervical cancer cells. Carcinogenesis 2011;32:1697-705.

9. Choi MJ, Park EJ, Min KJ, Park JW, Kwon TK. Endoplasmic reticulum stress mediates withaferin A-induced apoptosis in human renal carcinoma cells. Toxicol In Vitro 2011;25:692-8.

10. Oh JH, Lee TJ, Kim SH, Choi YH, Lee SH, Lee JM, et al. Induction of apoptosis by withaferin A in human leukemia U937 cells through down-regulation of Akt phosphorylation. Apoptosis 2008;13:1494-504.

11. Mandal C, Dutta A, Mallick A, Chandra S, Misra L, Sangwan RS, et 
al. Withaferin A induces apoptosis by activating p38 mitogen-activated protein kinase signaling cascade in leukemic cells of lymphoid and myeloid origin through mitochondrial death cascade. Apoptosis 2008;13:1450-64.

12. Amin AR, Kucuk O, Khuri FR, Shin DM. Perspectives for cancer prevention with natural compounds. J Clin Oncol 2009;27:2712-25.

13. Um HJ, Min KJ, Kim DE, Kwon TK. Withaferin A inhibits JAK/STAT3 signaling and induces apoptosis of human renal carcinoma Caki cells. Biochem Biophys Res Commun 2012;427:24-9.

14. Woo SM, Min KJ, Kim S, Park JW, Kim DE, Kim SH, et al. Axl is a novel target of withaferin $\mathrm{A}$ in the induction of apoptosis and the suppression of invasion. Biochem Biophys Res Commun 2014; 451:455-60.

15. Yco LP, Mocz G, Opoku-Ansah J, Bachmann AS. Withaferin A inhibits STAT3 and induces tumor cell death in neuroblastoma and multiple myeloma. Biochem Insights 2014;7:1-13.

16. Abubaker K, Luwor RB, Escalona R, McNally O, Quinn MA, Thompson EW, et al. Targeted disruption of the JAK2/STAT3 pathway in combination with systemic administration of paclitaxel inhibits the priming of ovarian cancer stem cells leading to a reduced tumor burden. Front Oncol 2014:4:75.

17. Corvinus FM, Orth C, Moriggl R, Tsareva SA, Wagner S, Pfitzner EB, et al. Persistent STAT3 activation in colon cancer is associated with enhanced cell proliferation and tumor growth. Neoplasia 2005:7:545-55.

18. Lin Q, Lai R, Chirieac LR, Li C, Thomazy VA, Grammatikakis I, et al. Constitutive activation of JAK3/STAT3 in colon carcinoma tumors and cell lines: inhibition of JAK3/STAT3 signaling induces apoptosis and cell cycle arrest of colon carcinoma cells. Am J Pathol 2005;167:969-80.

19. Aggarwal BB, Kunnumakkara AB, Harikumar KB, Gupta SR, Tharakan ST, Koca $C$, et al. Signal transducer and activator of transcription-3, inflammation, and cancer: how intimate is the relationship? Ann N Y Acad Sci 2009;1171:59-76.

20. Podolak I, Galanty A, Sobolewska D. Saponins as cytotoxic agents: a review. Phytochem Rev 2010;9:425-74

21. Yu ZY, Huang R, Xiao H, Sun WF, Shan YJ, Wang B, et al. Fluacrypyrim, a novel STAT3 activation inhibitor, induces cell cycle arrest and apoptosis in cancer cells harboring constitutively-active STAT3. Int J Cancer 2010;127:1259-70.

22. Zhao M, Jiang B, Gao FH. Small molecule inhibitors of STAT3 for cancer therapy. Curr Med Chem 2011;18:4012-8.

23. Lee MH, Kundu JK, Keum YS, Cho YY, Surh YJ, Choi BY. Resveratrol inhibits IL-6-induced transcriptional activity of AR and STAT3 in human prostate cancer LNCaP-FGC cells. Biomol
Ther (Seoul) 2014:22:426-30.

24. Amin H, Nayak D, Ur Rasool R, Chakraborty S, Kumar A, Yousuf $\mathrm{K}$, et al. Par-4 dependent modulation of cellular $\beta$-catenin by medicinal plant natural product derivative 3-azido Withaferin A [published online ahead of print May 12, 2015]. Mol Carcinog. doi: 10.1002/mc.22328.

25. Rai M, Jogee PS, Agarkar G, Santos CA. Anticancer activities of Withania somnifera: current research, formulations, and future perspectives [published online ahead of print April 7, 2015]. Pharm Biol. doi: 10.3109/13880209.2015.1027778.

26. Park JW, Min KJ, Kim DE, Kwon TK. Withaferin A induces apoptosis through the generation of thiol oxidation in human head and neck cancer cells. Int J Mol Med 2015;35:247-52.

27. Koduru S, Kumar R, Srinivasan S, Evers MB, Damodaran C. Notch-1 inhibition by Withaferin-A: a therapeutic target against colon carcinogenesis. Mol Cancer Ther 2010;9:202-10.

28. Nishikawa Y, Okuzaki D, Fukushima K, Mukai S, Ohno S, Ozaki Y, et al. Withaferin A induces cell death selectively in androgen- independent prostate cancer cells but not in normal fibroblast cells. PLoS One 2015;10:e0134137.

29. Heyninck K, Lahtela-Kakkonen M, Van der Veken P, Haegeman G, Vanden Berghe $\mathrm{W}$. Withaferin A inhibits NF-kappaB activation by targeting cysteine 179 in IKK $\beta$. Biochem Pharmacol 2014:91:501-9.

30. Rivat C, Rodrigues S, Bruyneel E, Piétu G, Robert A, Redeuilh G, et al. Implication of STAT3 signaling in human colonic cancer cells during intestinal trefoil factor 3 (TFF3): and vascular endothelial growth factor-mediated cellular invasion and tumor growth. Cancer Res 2005:65:195-202.

31. Lee J, Hahm ER, Singh SV. Withaferin A inhibits activation of signal transducer and activator of transcription 3 in human breast cancer cells. Carcinogenesis 2010;31:1991-8.

32. Min KJ, Choi K, Kwon TK. Withaferin A down-regulates lipopolysaccharide-induced cyclooxygenase-2 expression and PGE2 production through the inhibition of STAT1/3 activation in microglial cells. Int Immunopharmacol 2011;11:1137-42.

33. Li L, Shaw PE. A STAT3 dimer formed by inter-chain disulphide bridging during oxidative stress. Biochem Biophys Res Commun 2004:322:1005-11.

34. Becker AL, Orlotti NI, Folini M, Cavalieri F, Zelikin AN, Johnston AP, et al. Redox-active polymer microcapsules for the delivery of a survivin-specific siRNA in prostate cancer cells. ACS Nano 2011:5:1335-44

35. Slattery ML, Lundgreen A, Kadlubar SA, Bondurant KL, Wolff RK. JAK/STAT/SOCS-signaling pathway and colon and rectal cancer. Mol Carcinog 2013;52:155-66. 